\title{
MAPSSIC, a communicating MAPS-based intracerebral positrons probe for deep brain imaging in awake and freely-moving rats
}

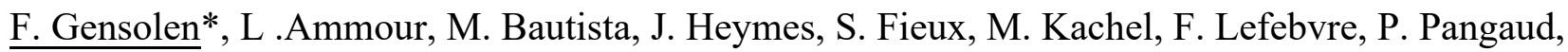 \\ L. Pinot, P. Lanièce, J. Baudot, P. Gisquet, L. Zimmer, M-A. Verdier, C. Morel \\ * Aix Marseille Univ, CNRS/IN2P3, CPPM, Marseille, France \\ gensolen@cppm.in2p3.fr
}

\begin{abstract}
Radioisotope imaging is a powerful tool to understand the biological mechanisms in-vivo, especially in the brain of small animals, providing a significant model to study the human brain.

In this context, we have developed and built a pixelated intracerebral positron probe to be embedded on awake and freely moving small animals, typically rats. This pixelated probe will represent a key instrument for neuroscientists to study neural mechanisms and correlate them to behavioral experiments.

We describe in this paper the simulations carried out to design the intracerebral sensor, its architecture, and the detection of positrons in a volume with a couple of sensors assembled back-toback. We also depict the architecture of the wireless acquisition system. Finally, we present the first measurements performed in real-time by this miniaturized probe with sealed radioactive sources and a ${ }^{18} \mathrm{~F}$ solution.
\end{abstract}

Index Terms - Active pixel sensors, Animal behavior, CMOS Image sensors, Implants, Molecular imaging, Monte Carlo methods, Nuclear Imaging, Positron emission tomography

\section{INTRODUCTION}

In the context of understanding the brain function, which is altered by diseases such as Parkinson, Alzeimer or by addictions, the correlation of neural activity measurements with behavioral experiments is of major interest for biologists. Small animals constitute preclinical models for this study. Therefore, we have started the development of a miniaturized and real-time intracerebral positrons probe for awake and

${ }^{1}$ This work was supported in part by the Project Instrumentation aux limites from CNRS in 2015 and 2017, in part by the France Life Imaging of the French Investissements d'Avenir program run by the Agence Nationale pour la Recherche under Grant FLI/ANR-11-INBS- 0006, and in part by IN2P3.

M. Bautista, F. Gensolen, P. Pangaud, and C. Morel are with the Aix Marseille Univ, CNRS/IN2P3, CPPM, Marseille, France.

L. Ammour, F. Lefebvre, L. Pinot, P. Laniece and M-A Verdier are with the Imagerie et Modélisation en Neurobiologie et Cancérologie (IMNC), CNRS, Univ Paris Sud, Université Paris-Saclay, F-91406 Orsay, France.

L. Ammour, F. Lefebvre, L. Pinot, P. Laniece and M-A Verdier are with Université de Paris, IMNC, F-91400 Orsay, France

J. Heymes, M. Kachel, and J. Baudot are with the Université de Strasbourg, CNRS, IPHC UMR 7178, Strasbourg, France.

S. Fieux and L. Zimmer are with the CERMEP-Imagerie du vivant, Université Claude Bernard Lyon 1, CNRS, INSERM, Hospices Civils de Lyon, Lyon, France.

P. Gisquet-Verrier is with the NeuroPSI CNRS/INSB, Univ Paris Sud, Orsay, France. freely moving small animals, typically rats. Taking advantage of the availability of new technologies and of the expertise we have acquired from former developments [1], [2], we propose a novel, robust, wireless, autonomous and lightweight active pixel positron probe.

We present in the following section the main results of the simulations carried out to validate the intracerebral sensor design with an emphasis on the modeling of the measurements with radioactive solutions (in particular ${ }^{18} \mathrm{~F}$ ).

Then we describe the architecture of the sensor and detection of positrons in a volume with the whole probe made of a couple of sensors assembled back-to-back. The wireless acquisition system is also described.

Finally, in the last section, we present the first measurements performed in real-time by this miniaturized probe and compare them to Monte Carlo simulations.

\section{MONTE CARLO SIMULATIONS OF THE SENSOR DESIGN}

In order to estimate the sensor performance regarding positron detection (referred to as direct detection in Fig 1) and rejection of annihilation photons and Compton electrons (referred to as indirect detection in Fig 1), we have performed Monte Carlo simulations using Gate [3] and studied the sensor performances including efficiency and sensitivity, and the deposited energy.

The pixel sensor circuit is developed in $180 \mathrm{~nm} \mathrm{CMOS}$ technology, with an $18 \mu \mathrm{m}$ thick high resistivity epitaxial layer sensitive to charged particles.

The sensor dimensions for the probe must take into account the biological, the electronics and the mechanical constraints. It results in a $12000 \times 610 \times 200 \mu \mathrm{m}^{3}$ sensor with a sensitive volume of $6400 \times 480 \times 190 \mu^{3}$. As shown in Fig 1 , this model was placed at the center of a cylindrical and homogeneous aqueous solution of ${ }^{18} \mathrm{~F},{ }^{15} \mathrm{O}$, or ${ }^{11} \mathrm{C}$. The simulations were carried out, without accounting for signal processing, nor charge diffusion. For more details about the Monte Carlo model of the sensor and the associated analysis, please refer to [4].

\section{A. Sensor efficiency}

The efficiency of the probe given by the count rate of the probe immersed in a ${ }^{18} \mathrm{~F}$ solution is illustrated in Fig 2, where isocontours at $10 \%$ and $1 \%$ are drawn. It appears that the efficiency of the probe to the detection of positrons is far greater than for the detection of $511 \mathrm{keV}$ annihilation photons 
and Compton electrons. This is what we expect from the sensor, which is designed to determine the local distribution of positron emitters in the brain. The volume bounded by the $1 \%$ efficiency isosurface is $(17.34 \pm 0.05) \mathrm{mm}^{3}$. This is in accordance to the volumes of interest for the rat brain structures, hippocampus, or caudate putamen, which are in the order of 40 and $30 \mathrm{~mm}^{3}$, respectively.

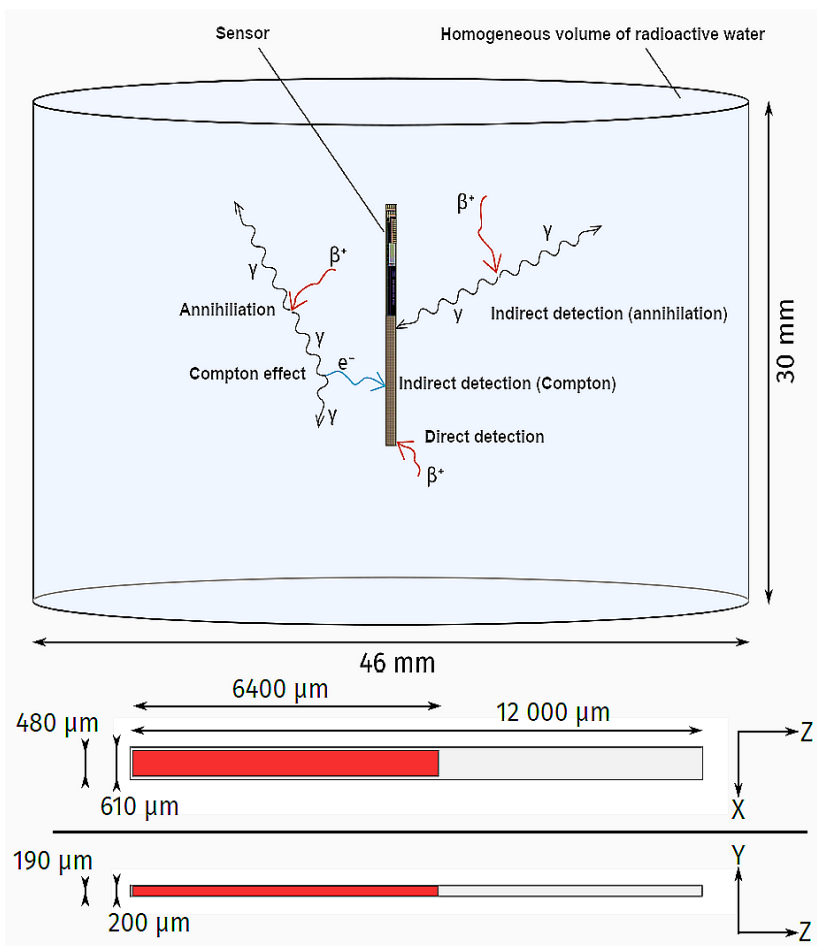

Fig 1. Sketch of the cylindrical radioactive solution (top) with the silicon sensor immersed in the center of the solution (bottom).

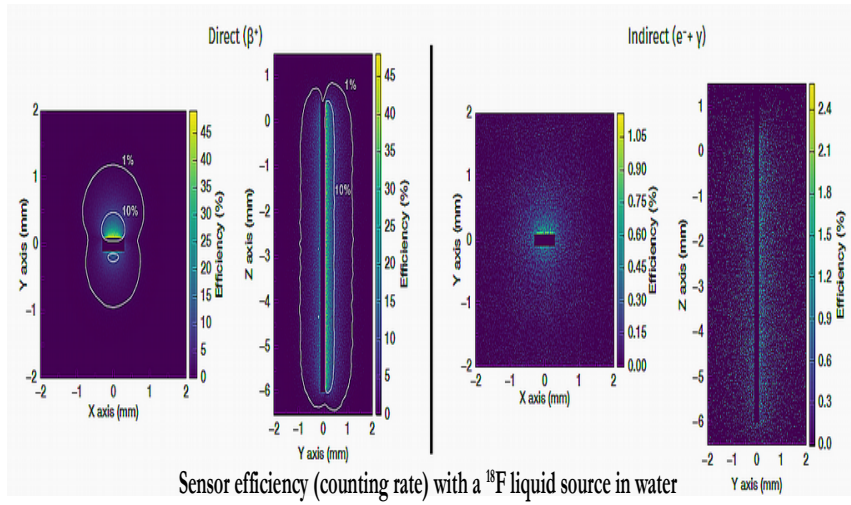

Fig 2. Spatial distribution of the efficiency given by the count rate of the sensor immersed in $a^{18} \mathrm{~F}$ water solution.

\section{B. Probe sensitivity}

The probe sensitivities to direct detection $S_{\text {direct }}$ with ${ }^{11} \mathrm{C},{ }^{15} \mathrm{O}$ and ${ }^{18} \mathrm{~F}$ are summarized in Table 1 . We observed a greater sensitivity to direct detection for ${ }^{11} \mathrm{C}$ and ${ }^{15} \mathrm{O}$ than for ${ }^{18} \mathrm{~F}$ solutions. This difference arises from the fact that positrons have a larger range in water for ${ }^{11} \mathrm{C}$ and ${ }^{15} \mathrm{O}$ than for ${ }^{18} \mathrm{~F}$. Nevertheless ${ }^{18} \mathrm{~F}$ is the most used isotope and has the best definition in terms of brain structure due to the lower range of its positrons. In addition sensitivity with ${ }^{18} \mathrm{~F}$ has been proven sufficient with the previous probe version [2]. Indirect detection sensitivity $S_{\text {indirect }}$ continuously increases because of the high penetration of $511 \mathrm{keV}$ annihilation $\gamma$-rays in water.

\begin{tabular}{l|ccc}
\hline Source & ${ }^{18} \mathrm{~F}$ & ${ }^{11} \mathrm{C}$ & ${ }^{15} \mathrm{O}$ \\
\hline$S_{\text {direct }}^{\max }\left(\mathrm{cps} \cdot \mathrm{Bq}^{-1} \cdot \mathrm{mm}^{3}\right)$ & $(8.83 \pm 0.01) \times 10^{-1}$ & $1.79 \pm 0.01$ & $4.34 \pm 0.01$ \\
\hline $50 \% S_{\text {direct }}^{\max }$ radius $(\mathrm{mm})$ & 0.50 & 0.77 & 1.6 \\
$99 \% S_{\text {direct }}^{\max }$ radius $(\mathrm{mm})$ & 1.73 & 2.85 & 5.96 \\
$99.9 \% S_{\text {direct }}^{\max }$ radius $(\mathrm{mm})$ & 2.12 & 3.53 & 7.36 \\
\hline$S_{\text {direct }}=20$ radius $(\mathrm{mm})$ & 1.53 & 2.96 & 5.70 \\
$S_{\text {indirect }}$ & 3.55 & 7.48 & 20.3 \\
$\frac{S_{\text {direct }}}{S_{\text {indirect }}}=10$ radius $(\mathrm{mm})$ & 8.28 & 20.8 & $>23$ \\
$\frac{S_{\text {direct }}}{S_{\text {indirect }}}=5$ radius $(\mathrm{mm})$ & & & \\
\hline
\end{tabular}

Table 1. Maximum direct detection sensitivity values, radii for a given sensitivity value, and radii for different ratio of direct detection sensitivity over indirect detection sensitivity.

With a ${ }^{18} \mathrm{~F}$ source of $5 \mathrm{~mm}$ radius, we get a $S_{\text {direct }}$ over $S_{\text {indirect }}$ ratio of $8,28 \pm 0,01$, which also make this isotope the most interesting for our application.

\section{Deposited energy spectra}

The CMOS monolithic active pixel sensor (MAPS) features $16 \times 128$ pixels of $30 \times 50 \mu \mathrm{m}^{2}$. The spectra of deposited energies by positrons, electrons and photons are presented in Fig 3. Deposited energies are maximum for $6.9 \mathrm{keV}$ and $7.9 \mathrm{keV}$ for positrons and electrons respectively, while $511 \mathrm{keV} \gamma$-rays involve relatively lower energy depositions.

Table 2 summarizes the deposited energy distribution for direct detection. This confirms that the MAPS with a detection threshold in the order of few $\mathrm{keV}$ can be suitable for direct detection of positrons. 


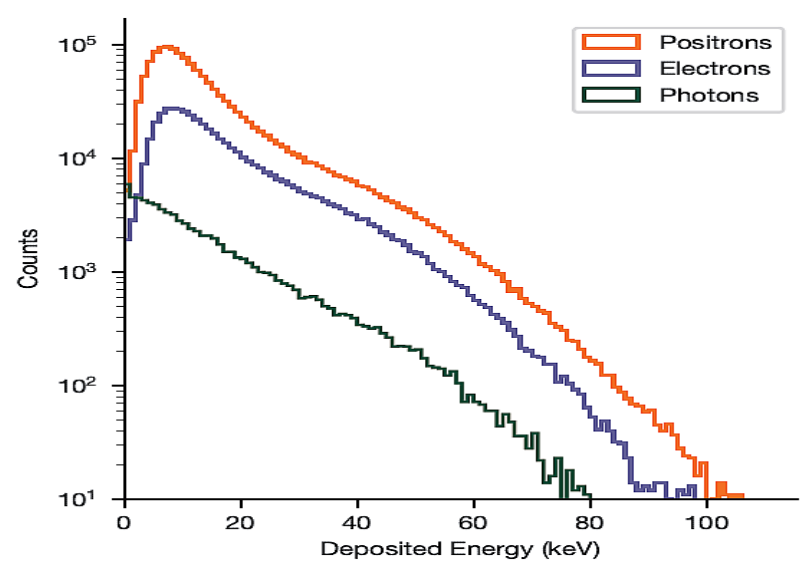

Fig 3. Deposited energy spectra for $16 \times 128$ pixels $30 \times 50 \mu \mathrm{m}^{2}$ immersed in a homogeneous ${ }^{18} \mathrm{~F}$ solution. In case multiple pixels are hit in the same event, the highest deposited energy in one pixel is considered.

\begin{tabular}{|l|ccc|}
\hline Isotopes & ${ }^{18} \mathrm{~F}$ & ${ }^{11} \mathrm{C}$ & ${ }^{15} \mathrm{O}$ \\
\hline$E_{\text {peak }}(\mathrm{keV})$ & 6.9 & 7.2 & 6.1 \\
$E_{\text {median }}(\mathrm{keV})$ & 11.0 & 9.51 & 8.72 \\
$E_{\text {mean }}(\mathrm{keV})$ & 15.1 & 12.9 & 11.4 \\
fraction $<1 \mathrm{keV}$ & $0.37 \%$ & $0.44 \%$ & $0.59 \%$ \\
fraction $<10 \mathrm{keV}$ & $44.3 \%$ & $52.9 \%$ & $59.0 \%$ \\
\hline
\end{tabular}

Table 2. Peak, median, and mean deposited energy in the pixels for direct detection only. Sensor immersed in a homogeneous ${ }^{18} \mathrm{~F}$ solution.

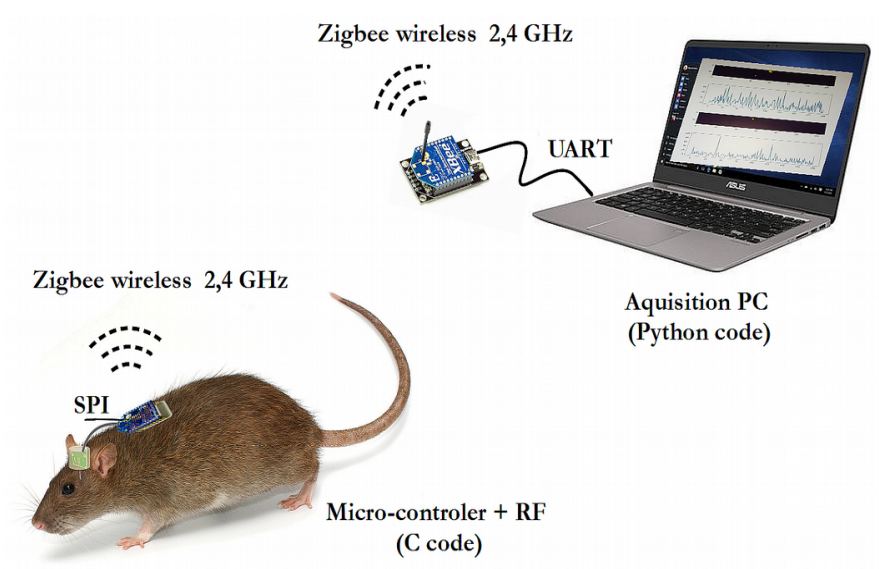

Fig 4: Artistic view of the whole acquisition system.

\section{MAPSSIC PROBE DESIGN AND SETUP}

At this point the positron sensor has been specified and modeled by simulations. We had then to build a wireless, lightweight, robust, autonomous, biocompatible and reliable acquisition system. For this, we realized the MAPS CMOS sensor circuit, then assembled a couple of these sensors backto-back to constitute the probe, and designed a hybrid flex- rigid printed circuit board (PCB) to interface the probe with the embedded control and communication electronics. Finally, we coated the probe to make it biocompatible. The following sections detail all the design and manufacturing steps of the setup. Fig 4 shows the whole acquisition system.

\section{A. MAPS CMOS circuit}

Our positron sensor has been designed in a $0.18 \mu \mathrm{m}$ CMOS technology on $18 \mu \mathrm{m}$ thick high resistivity $(>1 \mathrm{k} \Omega \cdot \mathrm{cm})$ epitaxial layer. It is composed of $30 \times 50 \mu \mathrm{m}^{2}$ pixels arranged in a $16 \times 128$ matrix and has a power consumption lower than $55 \mathrm{nW} /$ pixel [5]. The pixel readout is a rolling shutter scheme with an asynchronous memorization of the hit in the pixel (1 bit). Each pixel is reset right after it has been readout. The control of the pixel analog biasing and configuration (DACs values) is performed via SPI (Serial Peripheral Interface) protocol.

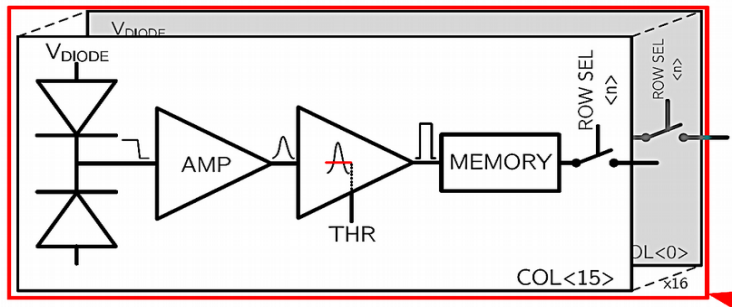

Fig 5: Architecture of a pixel.

\section{B. $3 D$ positron sensing and biocompatible coating}

The positrons are detected mainly on the epitaxial layer side of the sensor. In order to build a probe able to detect the positrons on both sides while increasing the sensitivity of the probe as well as its robustness, we decided to assemble a couple of sensors back-to-back, as shown on Fig 6.

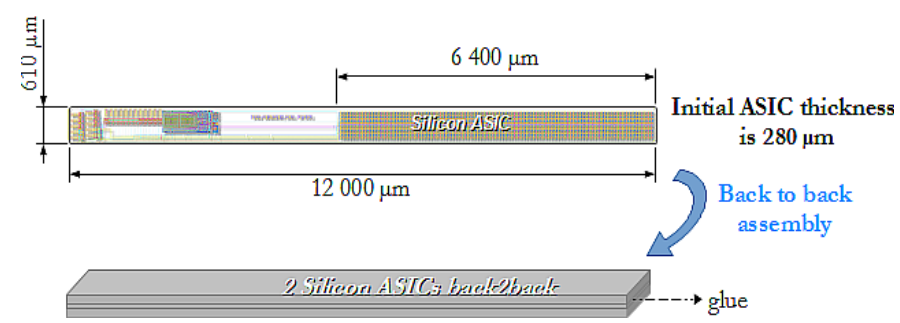

Fig 6: Dimensions and geometrical view of the MAPS probe.

Back-to-back assembly of a couple of MAPS probes wirebonded to a thin hybrid flex-rigid PCB resulted in 3D devices featuring various thicknesses of 400, 500 and $560 \mu \mathrm{m}$.

An additional $6 \mu \mathrm{m}$ biocompatible coating of parylene was then deposited over the whole probe surface to allow for its implantation in the brains (Fig 7). 


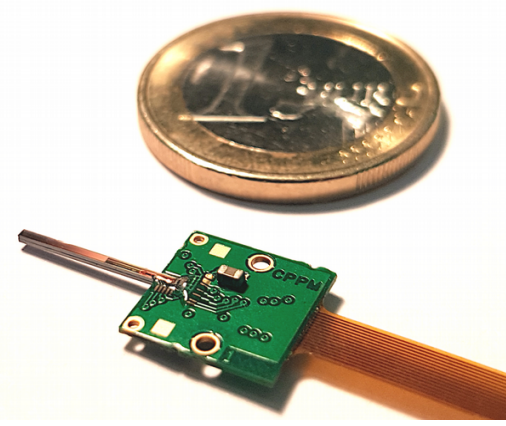

Silicon ASICs mounted and wire-bonded

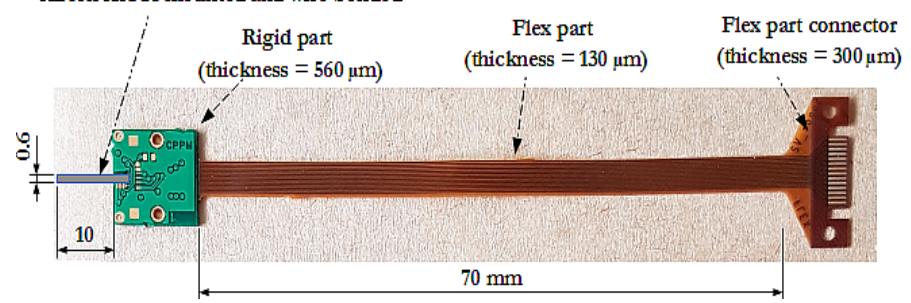

Fig 7: Back-to-back sensors assembly mounted and wirebonded on an interface $P C B$ in hybrid flex-rigid $P C B$ process.
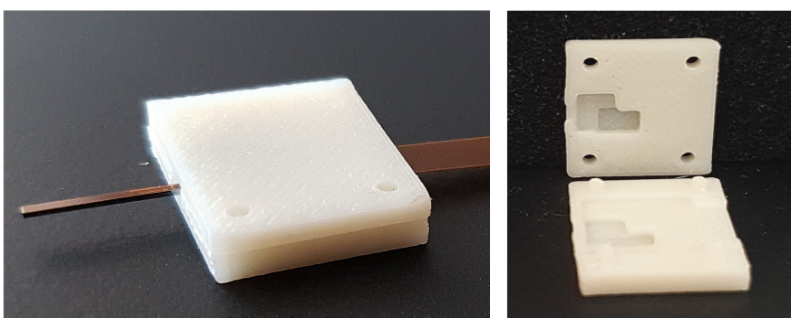

Fig 8: Encapsulated probe PCB interface.

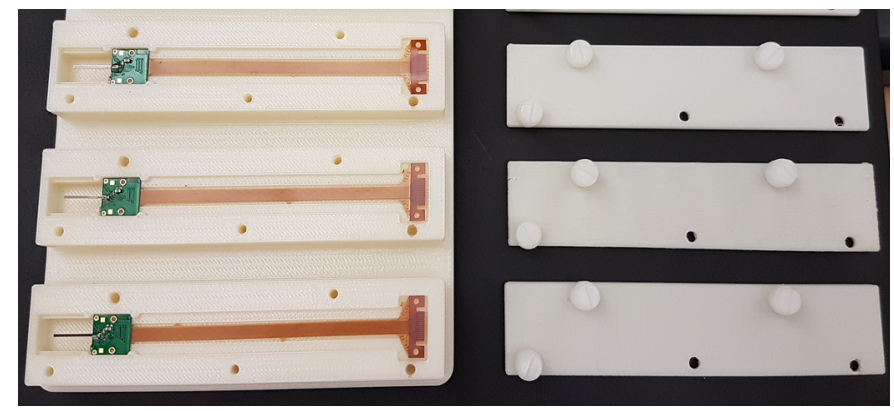

Fig 9: 3D-printing tool designed for the protection and safe transportation of the probes.

\section{Wireless embedded electronics}

As described in Fig 4, the control of the probe is carried out by a computer, which communicates with an embedded electronics through the Zigbee $2.4 \mathrm{GHz}$ wireless protocol. This radio communication is performed via2two radiofrequency Xbee S2C modules from Digi-International. These modules are very well suited for our application since each module embeds two micro-controllers. One micro-controller is programmable and therefore set up to control the probe and readout the pixels, the other one is dedicated to the RF communication.

A battery and a power management is also embedded on the animal to provide energy. All the embedded electronics weights less than $20 \mathrm{~g}$.

The micro-controller program is written in $\mathrm{C}$ for the implementation of low level instructions. The computer software has been developed in Python for the Human Machine Interface in order to control the probe and manage data handling.

\section{MEASUREMENTS RESUltS}

To validate experimentally the probe, we have measured the counting rate of the probe with different sealed radioactive sources and its sensitivity in a radioactive solution.

\section{A. Radioactive setup}

Fig 10 bellow describes the setup for measurements with the probe immersed in a ${ }^{18} \mathrm{~F}$ water solution. The probe flex connector is plugged in the corresponding male part with an interface board connected to the micro-controller PCB.
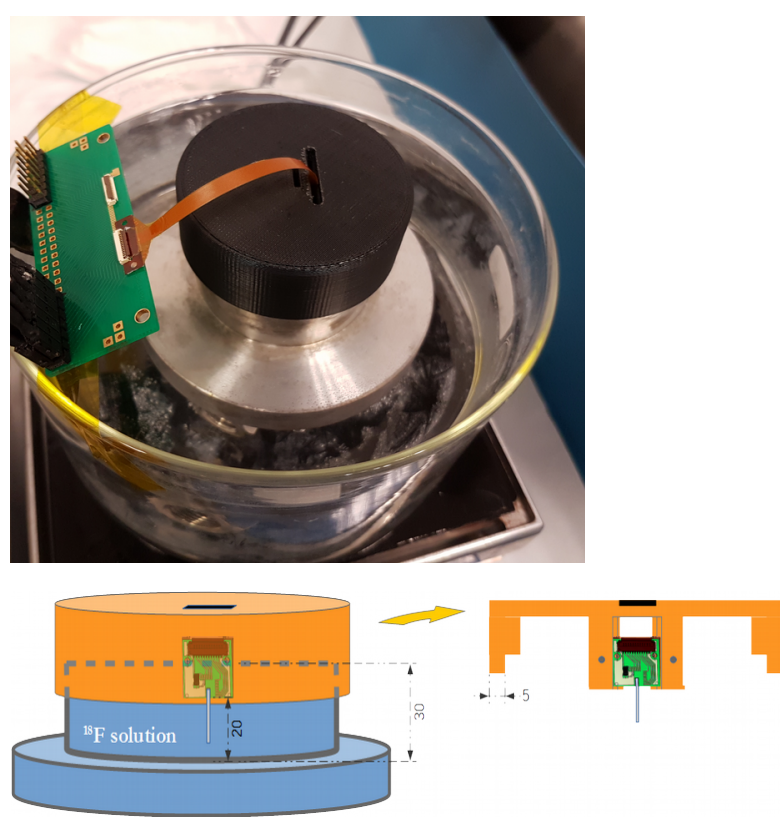

Fig 10: Experimental setup with the probe immersed in $a^{18} \mathrm{~F}$ water solution.

In the case of measurements with sealed radioactive sources, we used a trusquin to place the sources at a given and stable distance from the probe in its transportation tool (Fig 9). 


\section{B. Results}

Fig 11 reports some examples of frames acquisitions with different types of radioactive sources irradiating a single sensor.

With a pixel integration time of $224 \mathrm{~ms}$, we measured a dark noise of $5.5 \times 10^{-3} \mathrm{cps} / \mathrm{mm}^{2}$ with a few noisy pixels removed. The mean pixel cluster size (typical for beta particles interactions) was 3,87 pixels.

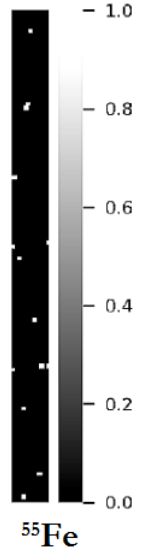

X-ray
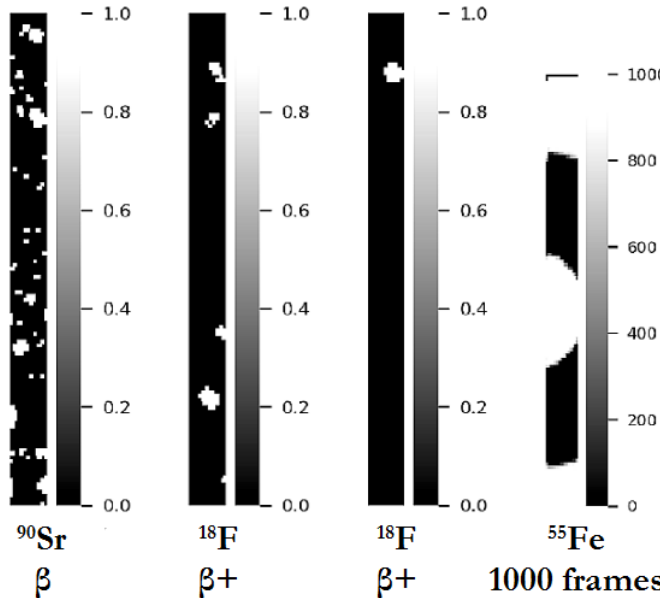

Fig 11: Examples of acquisitions with different types of radioactive sources.

We have also immersed the probe in ${ }^{18} \mathrm{~F}$ water solution and measured a source decay half-life of $(110.7 \pm 0.8) \mathrm{min}$., thus assessing the probe linearity (Fig 12).

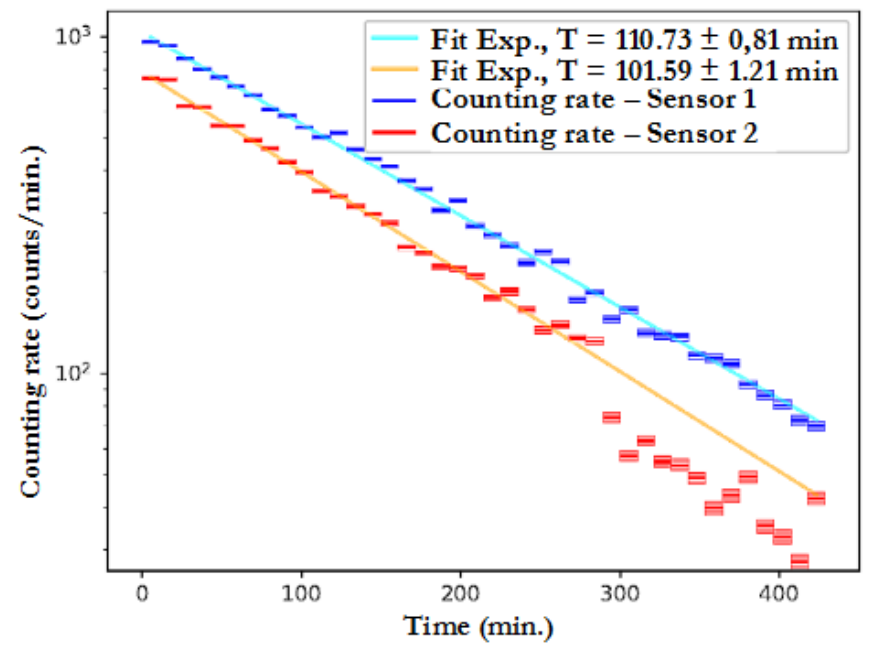

Fig 12: Probe count rate vs. activitv.

However we experienced important instabilities leading to strong disparities in the count rate from one probe to the other.
Also the experimental sensitivity measured is $8.9 \times 10^{-2} \mathrm{cps} . \mathrm{Bq}^{-1} \cdot \mathrm{mm}^{3}$, to be compared to the simulated one which was $(1.10 \pm 0.02) \mathrm{cps} . \mathrm{Bq}^{-1} \cdot \mathrm{mm}^{3}$.

These two surprising results are possibly explained by a problem on the sensor digital inputs/outputs pads, which suffer from a very important leakage current (tens of $\mathrm{mA}$ ). This resulted in increasing the temperature of the sensor up to $60{ }^{\circ} \mathrm{C}$, which affected the performances of the chip.

A new version of the MAPS CMOS circuit is currently under design to correct this problem.

\section{CONCLUSION}

Behavioral neuro-imaging is required to address the limits of anesthesia model and to correlate neuro-imaging with behavioral studies. In that context we developed MAPSSIC, the first CMOS active pixel sensor device for real-time positron imaging of freely moving small animals.

Monte Carlo simulation studies were performed to design the probe, which was then fabricated together with the associated wireless control and acquisition system.

Measurements carried out with sealed sources and also with the probe immersed in a radioactive solution have demonstrated that MAPSSIC can provide a robust tool for invivo imaging.

The short terms actions are the design of a new CMOS circuit to correct for high current on digital pads problem encountered with the first version of the probe that resulted in a significant probe temperature elevation.

\section{REFERENCES}

[1] F. Pain et al., "SIC: an intracerebral radiosensitive probe for in vivo neuropharmacology investigations in small laboratory animals: prototype design, characterization, and in vivo evaluation." IEEE Transactions on Nuclear Science, vol. 49, no. 3, pp.822-826, 2002.

[2] L. Balasse et al., "PIXSIC: A Wireless Intracerebral Radiosensitive Probe in Freely Moving Rats." Molecular Imaging, vol. 14, no. 43, pp. 484-489, 2015.

[3] S. Jan et al., "GATE V6: a major enhancement of the GATE simulation platform enabling modelling of CT and radiotherapy". Phys. Med. Biol., vol. 54 , no. 4 , pp. $881,2011$.

[4] L. Ammour et al., "MAPSSIC, a Novel CMOS Intracerebral Positrons Probe for Deep Brain Imaging in Awake and Freely Moving Rats: A Monte Carlo Study." IEEE Transactions on Radiation and Plasma Medical Sciences, vol. 3, no. 3, pp. 302-314, 2019.

[5] J. Heymes et al., "Implantable CMOS pixel sensor for positron imaging in rat brain." Nucl. Instrum. Meth. A, vol., 911, pp. 19-24, 2018. 